\title{
Sparse phase retrieval of structured signals by Prony's method
}

\author{
Robert Beinert ${ }^{1}$ and Gerlind Plonka ${ }^{2}$ \\ ${ }^{1}$ Institut für Mathematik und Wissenschaftliches Rechnen \\ KARL-Franzens-Universität Graz \\ Heinrichstraße 36 \\ 8010 Graz, Austria \\ ${ }^{2}$ Institut für Numerische und Angewandte Mathematik \\ Georg-August-Universität Göttingen \\ Lotzestraße 16-18 \\ 37083 Göttingen, Germany \\ 9 May 2017 \\ Correspondence \\ R. BEINERT: robert.beinert@uni-graz.at \\ G. PLonka: plonka@math.uni-goettingen.de
}

\begin{abstract}
The phase retrieval problem consists in the recovery of a complex-valued signal from the magnitudes of its Fourier transform. Restricting ourselves to the case of sparse structured signals $f$, which can be represented as a linear combination of $N$ arbitrary translations of a given generator function, we show that almost all $f$ can be recovered from $\mathcal{O}\left(N^{2}\right)$ intensity measurements $|\mathcal{F}[f](\omega)|$ up to trivial ambiguities.
\end{abstract}

Keywords. $\quad$ sparse phase retrieval, sparse structured signals, Prony's method AMS subject classification. $\quad 42 \mathrm{~A} 05,94 \mathrm{Ao} 8,94 \mathrm{~A} 12$

\section{Introduction}

Phase retrieval means that we wish to recover an unknown signal from its Fourier intensity, i.e., from the absolute value of its Fourier transform. Recovery problems of this kind appear in a wide range of applications such as in electron microscopy, wave front sensing, laser optics [1, 2] as well as in X-ray crystallography and speckle imaging [3]. Due to the loss of the phase, in general, the problem is ill-posed and cannot be solved uniquely. Here we restrict ourselves to the recovery of one-dimensional sparse structured signals of the form

$$
f(t):=\sum_{j=1}^{N} c_{j} \phi\left(t-T_{j}\right)
$$


where $c_{j} \in \mathbb{C}$, and where $\phi$ is a given generator function in $L^{1}$, from their continuoustime Fourier intensities

$$
|\mathcal{F}[f](\omega)|:=|\widehat{f}(\omega)|:=\left|\int_{-\infty}^{\infty} f(t) \mathrm{e}^{-\mathrm{i} \omega t} \mathrm{~d} t\right|=|\widehat{\phi}(\omega)|\left|\sum_{j=1}^{N} c_{j} \mathrm{e}^{-\mathrm{i} \omega T_{j}}\right| \quad(\omega \in \mathbb{R}) .
$$

We assume that $\phi$ satisfies $\phi(-t)=\overline{\phi(t)}$. Since the Fourier intensity is not affected by rotations $\mathrm{e}^{\mathrm{i} \alpha} f$ with $\alpha \in \mathbb{R}$, time shifts $f\left(\cdot-t_{0}\right)$ with $t_{0} \in \mathbb{R}$, and conjugate reflections $\overline{f(-\cdot)}$ of the true signal $f$, we cannot expect to solve the phase retrieval problem up to these trivial ambiguities.

\section{Uniqueness for structured signals}

Besides the trivial ambiguities, the phase retrieval problem usually possesses further non-trivial ambiguities, which may heavily differ from the true solution $f$, see for instance $[4,5,6]$. However, if the signal $f$ is of the form (1), than the non-trivial ambiguities of the phase retrieval problem can be entirely avoided under certain assumptions on the knots $T_{j}$ and coefficients $c_{j}$ of the signal. In order to derive these assumptions and to guarantee uniqueness of the recovery of $f$ (up to the trivial ambiguities presented above), we follow the approach in [7], which is closely related to our setting.

Theorem 2.1. Let $f$ be a signal of the form (1), whose knot differences $T_{j}-T_{k}$ differ pairwise for $j, k \in\{1, \ldots, N\}$ with $j \neq k$, and whose coefficients satisfy $\left|c_{1}\right| \neq\left|c_{N}\right|$. Further, let $h$ be a step size such that $h\left(T_{j}-T_{k}\right) \in(-\pi, \pi)$ for all $j, k$, and such that $\mathscr{F}[\phi](h \ell) \neq 0$ for $\ell=0, \ldots, 3 / 2 N(N-1)$. Then $f$ can be uniquely recovered from its Fourier intensities $|\mathcal{F}[f](h \ell)|$ with $\ell=0, \ldots, 3 / 2 N(N-1)$ up to trivial ambiguities.

Proof. Applying the given Fourier intensity $|\widehat{f}(\omega)|$, we consider the exponential sum

$$
P(\omega):=\frac{|\widehat{f}(\omega)|^{2}}{|\widehat{\phi}(\omega)|^{2}}=\sum_{j=1}^{N} \sum_{k=1}^{N} c_{j} \bar{c}_{k} \mathrm{e}^{-\mathrm{i} \omega\left(T_{j}-T_{k}\right)}=\sum_{\ell=-N(N-1) / 2}^{N(N-1) / 2} \gamma_{\ell} \mathrm{e}^{-\mathrm{i} \omega \tau_{\ell}} .
$$

Using the assumption that the differences $T_{j}-T_{k}$ differ pairwise for $j \neq k$, we have rearranged the terms of the sum (2) such that the distinct non-zero frequencies $\tau_{\ell}:=T_{j}-T_{k}$ together with the zero frequency $\tau_{0}:=0$ are ordered by size. The $N(N-1)+1$ frequency differences $\tau_{\ell}$ for $\ell=-N(N-1) / 2, \ldots, N(N-1) / 2$ are obviously symmetric, i.e. $\tau_{-\ell}=\tau_{\ell}$. The corresponding coefficients in (2) are now given by $\gamma_{\ell}:=c_{j} \bar{c}_{k}$ and $\gamma_{0}:=\sum_{j=1}^{N}\left|c_{j}\right|^{2}$ and fulfil the conjugate symmetry $\gamma_{-\ell}=\bar{\gamma}_{\ell}$. Note that the mapping $(j, k) \mapsto \ell$ is unknown and also has to be recovered. In a first step, we apply an adapted version of Prony's method [7], to determine all frequency differences $\tau_{\ell}$ and the corresponding coefficients 
$\gamma_{\ell}$ of (2) from the equispaced samples $P(h \ell)$ with $\ell=0, \ldots, 3 / 2 N(N-1)$, where $h$ is a suitable step size such that $h\left(T_{j}-T_{k}\right) \in(-\pi, \pi)$ for all $j, k$, and such that $\mathscr{F}[\phi](h \ell) \neq 0$. In the second step, we use the frequency differences and the corresponding coefficients to recover the parameters $T_{j}$ and $c_{j}$ of (1). For this purpose, we denote the list of all positive frequency differences by $\mathcal{T}:=\left\{\tau_{\ell}\right\}_{\ell=1}^{N(N-1) / 2}$. Obviously, the maximal distance $\tau_{N(N-1) / 2}$ corresponds to $T_{N}-T_{1}$. Considering the trivial shift ambiguity, we can thus assume that $T_{1}=0$ and $T_{N}=\tau_{N(N-1) / 2}$. Next, the second largest distance $\tau_{(N(N-1) / 2)-1}$ is either $T_{N-1}-T_{1}$ or $T_{N}-T_{2}$. We can now fix $T_{N-1}=T_{N-1}-T_{1}=\tau_{(N(N-1) / 2)-1}$ thereby suppressing the trivial reflection and conjugation ambiguity. Further, the difference set $\mathcal{T}$ has to contain a difference $\tau_{\ell^{*}}$ such that $\tau_{\ell^{*}}+\tau_{(N(N-1) / 2)-1}=\tau_{N(N-1) / 2}$, which implies that $\tau_{\ell^{*}}=T_{N}-T_{N-1}$. Considering the related coefficients in (2), we obtain

$$
c_{N} \bar{c}_{1}=\gamma_{N(N-1) / 2}, \quad c_{N-1} \bar{c}_{1}=\gamma_{(N(N-1) / 2)-1} \quad \text { and } \quad c_{N} \bar{c}_{N-1}=\gamma_{\ell^{*}} .
$$

Up to a global phase factor, the solution of this system is given by

$$
c_{1}=\sqrt{\left|\frac{\gamma_{N(N-1) / 2} \overline{\gamma_{(N(N-1) / 2)-1}}}{\gamma_{\ell^{*}}}\right|}, \quad c_{N-1}=\frac{\gamma_{(N(N-1) / 2)-1}}{\bar{c}_{1}} \quad \text { and } \quad c_{N}=\frac{\gamma_{N(N-1) / 2}}{\bar{c}_{1}} .
$$

On the basis of the recovered knots $T_{1}, T_{N-1}, T_{N}$ and coefficients $c_{0}, c_{N-1}, c_{N}$, we proceed with the third largest distance $\tau_{(N(N-1) / 2)-2}$. As before, there exists a difference $\tau_{\ell^{*}} \in \mathcal{T}$ such that $\tau_{(N(N-1) / 2)-2}+\tau_{\ell^{*}}=\tau_{N(N-1) / 2}$. Now, there are only two possibilities: either $\tau_{(N(N-1) / 2)-2}=T_{N}-T_{2}$ or $\tau_{(N(N-1) / 2)-2}=T_{N-2}-T_{1}$, which results in the related coefficients

$$
c_{2}=\frac{\gamma_{\ell^{*}}}{\bar{c}_{1}}=\frac{\bar{\gamma}_{(N(N-1) / 2)-2}}{\bar{c}_{N}} \quad \text { or } \quad c_{N-2}=\frac{\bar{\gamma}_{\ell^{*}}}{\bar{c}_{N}}=\frac{\gamma_{(N(N-1) / 2)-2}}{\bar{c}_{1}}
$$

respectively. If only one of the equations in (3) holds true, we can either recover $T_{2}$ with $c_{2}$ or $T_{N-2}$ with $c_{N-2}$. On the other side, if both equations in (3) hold true, we obtain

$$
\left|\frac{c_{N}}{c_{1}}\right|=\left|\frac{\gamma_{(N(N-1) / 2)-2}}{\gamma_{\ell^{*}}}\right|=\left|\frac{c_{1}}{c_{N}}\right|
$$

which contradicts the assumption $\left|c_{N}\right| \neq\left|c_{1}\right|$. Removing the frequency differences $\tau_{\ell}$ that correspond to the differences $T_{j}-T_{k}$ of the determined knots from the set $\mathcal{T}$, we can inductively repeat this idea to recover the remaining knots and coefficients of the true signal $f$.

Identifying the space of structured signals (1) with the real space $\mathbb{R}^{3 N}$, we notice that the condition $T_{j_{1}}-T_{k_{1}}=T_{j_{2}}-T_{k_{2}}$ for fixed indices $j_{1}, j_{2}, k_{1}$, and $k_{2}$ defines a hyperplane. Since a similar observation holds for $\left|c_{1}\right|=\left|c_{N}\right|$, the signals excluded in Theorem 2.1 form a set of Lebesgue measure zero. 
Corollary 2.2. Almost all signals $f$ in (1) can be uniquely recovered from their Fourier intensity $|\mathcal{F}[f]|$ up to trivial ambiguities.

Remark 2.3. Due to the constructive character of the proof of Theorem 2.1, we can recover an unknown structured signal (1) from its Fourier intensity analytically as well as numerically. The assumption of Theorem 2.1 can directly be verified during the recovery. If the assumption that the differences $T_{j}-T_{k}$ are pairwise distinct is violated, Prony's method yields less than $N(N-1)+1$ distinct frequencies $\tau_{\ell}$. The assumption $\left|c_{1}\right| \neq\left|c_{N}\right|$ can be verified in the beginning of the second recovery step, where $c_{1}, c_{N-1}$, and $c_{N}$ are computed.

Acknowledgements. The first author gratefully acknowledges the funding of this work by the Austrian Science Fund (FWF) within the project $\mathrm{P} 28858$, and the second author the funding by the German Research Foundation (DFG) within the project PL 170/16-1. The Institute of Mathematics and Scientific Computing of the University of Graz, with which the first author is affiliated, is a member of NAWI Graz (http://www. nawigraz.at/).

\section{References}

[1] B. Seifert, H. Stolz, and M. Tasche, J. Opt. Soc. Am. B 21(5), 1089-1097 (2004).

[2] B. Seifert, H. Stolz, M. Donatelli, D. Langemann, and M. Tasche, J. Phys. A: Math. and Gen. 39(16), 4191-4206 (2006).

[3] J. Ranieri, A. Chebira, Y. M. Lu, and M. Vetterli, Phase retrieval for sparse signals: uniqueness conditions, arXiv:1308.3058v2, 2013.

[4] R. Beinert and G. Plonka, J. Fourier Anal. Appl. 21(6), 1169-1198 (2015).

[5] R. Beinert and G. Plonka, PAMM, Proc. Appl. Math. Mech. 15(1), 653-654 (2015).

[6] R. Beinert, Inf. Inference (2016), DOI 10.1093/imaiai/iawo18.

[7] R. Beinert and G. Plonka, Front. Appl. Math. Stat 3(5) (2017). 